\title{
On the units of a modular group ring II K.R. Pearson
}

\begin{abstract}
Let $R$ be a ring of nonzero characteristic and let $G$ be a finite group with subgroup $H$. It is shown that $H$ is a normal subgroup of the group of units of the group ring $R G$ if and only if $H$ is contained in the centre of $G$ or $R$ is the field with 2 elements, $G$ is the symmetric group on 3 letters and $H$ is normal in $G$.
\end{abstract}

Let $R$ be a ring of nonzero characteristic and with identity 1 , let $G$ be a finite group with a subgroup $H$. We examine when $\{1 h \mid h \in H\}$, which we again denote by $H$, is a normal subgroup of the group $(R G)$ * of units of the group ring $R G$, and prove the following theorem. $\left(z_{n}\right.$ denotes the ring of rational integers modulo $n$ and $S_{3}$ and $A_{3}$ denote the symmetric and alternating groups on 3 letters.)

THEOREM. Let $R$ be a ring of nonzero characteristic and let $G$ be a finite group with a subgroup $H$. Then $H$ is a normal subgroup of the group of wits of the group ring $R G$ if and only if $H$ is contained in the centre of $G$ or $R \simeq Z_{2}, G \simeq S_{3}$ and $H \simeq S_{3}$ or $A_{3}$.

This extends Theorem 1 of [4] in two directions. In the first place $R$ was restricted there to $Z_{n}$; the present theorem now gives a complete characterization of those group rings $R G$ with $R$ of nonzero characteristic which satisfy the Condition III stated in [4]. Secondly, in [4] only the case $H=G$ was considered. Although Theorem 1 of [4] is a special 
case of the present theorem, the proof given here makes essential use of it and does not subsume it.

The removal of the restriction $H=G$ was suggested by Eldridge [2] where it is proved that if $G$ is a locally finite $p$-group and $H$ is a subgroup of $G$, then $H$ is normal in $\left(z_{p} G\right)^{*}$ if and only if $H$ is contained in the centre of $G$.

\section{Outline of the proof}

If $H$ is contained in the centre of $G$ then $H$ is contained in the centre of $R G$ and clearly $H \triangleleft(R G)^{*}$. Also it is shown in Lemma 7 of [4] that $S_{3} \triangleleft\left(z_{2} S_{3}\right) *$, and it follows easily from this result that $A_{3} \triangleleft\left(z_{2} S_{3}\right)^{*}$. This takes care of the sufficiency part of the theorem.

We now consider the necessity part, and first show that it is sufficient to prove the theorem in the case where $R=Z_{p}$ for some prime $p$. To do this let us assume the result when $R \simeq z_{p}$ and consider an arbitrary ring $R$ of nonzero characteristic $n$ with $H \triangleleft(R G)^{*}$. If $R_{0}$ is the prime subring of $R$ then: $H \triangleleft\left(R_{0} G\right)^{*}$. Also if $p$ is a prime dividing $n$ the natural homomorphism from $z_{n}$ to $z_{p}$ extends to a homomorphism from $Z_{n} G$ to $Z_{p} G$. Since $Z_{n} G$ is finite, it follows from Theorem 3 of [4] that $H \triangleleft\left(z_{p} G\right)^{*}$. Thus either $H$ is contained in the centre of $G$ or $p=2, G \simeq S_{3}$ and $H \simeq S_{3}$ or $A_{3}$. This means that either $H$ is contained in the centre of $G$, and we are finished, or $n=2^{k}$ and we can take $A_{3} \subseteq H \subseteq G=S_{3}$. The following lemma gives the required result.

LEMMA 1. Let $R$ be a ring of characteristic $2^{k}$ with $k \geq 1$. If $A_{3} \subseteq H \subseteq G=S_{3}$ and $H \triangleleft(R G)^{*}$ then $R=Z_{2}$.

Proof. Let $S_{3}=\left\langle a, b \mid a^{2}=b^{3}=1, b a=a b^{2}\right\rangle$. Suppose, if possible, that $k>1$. Then if $x=2^{k-1}$ we have $x^{2}=0=2 x$ and so 
$(1+x a)^{2}=1$. But

$$
(1+x a) b(1+x a)=b+x a b+x a b^{2},
$$

which is not in $H$. Hence $k=1$. If $\theta=(1+a)\left(b+b^{2}\right)$, then $\theta^{2}=0$. Thus if $y \in R, 1+y \theta$ is its own inverse. But

$$
(1+y \theta) b(1+y \theta)=\left(1+y^{2}\right) b+y^{2} b^{2}+\left(y^{2}+y\right)\left(a b+a b^{2}\right),
$$

which is in $H$ only if $y=0$ or 1 . Therefore $R \simeq z_{2}$ as required.

Accordingly in what follows we always assume that $H \triangleleft\left(z_{p} G\right) *$. We show that either $H$ is contained in the centre of $G$ or $p=2, G \simeq S_{3}$ and $H \simeq S_{3}$ or $A_{3}$. Because $\left(z_{p} H\right) *$ is a subgroup of $\left(z_{p} G\right) *$ we know that $H \triangleleft\left(z_{p} H\right)^{*}$ and can apply Theorem 1 of [4] to see that either $H$ is abelian or $p=2$ and $H \simeq S_{3}$. When $p$ does not divide $|G|$ the result is proved in $\$ 2$. When $p$ divides $|G|$ the result is proved in $\$ 3$ and $\$ 4$ for the cases $p \geq 3$ and $p=2$ respectively.

One simple fact we use frequently is that $H$ is normal in any subgroup of $\left(2_{p} G\right)^{*}$. In particular $H$ is normal in $G$ and, if $L$ is any subgroup of $G$ containing $H, H \diamond\left(z_{p} L\right)^{*}$.

\section{The semisimple case}

LEMMA 2. If $p$ does not divide $|G|$ then $H$ is contained in the centre of $G$.

Proof. Suppose, if possible, that $H$ is not contained in the centre of $G \cdot Z_{p} G$ is semisimple and there must exist a central idempotent $e$ in $Z_{p} G$ such that $H e$ is not contained in the centre of $\left(z_{p} G\right) e$ and $\left(z_{p} G\right) e \simeq M_{n}\left(G F\left(p^{k}\right)\right)$ for some $n \geq 2, k \geq 1$. Let

$\theta:\left[\left(z_{p} G\right) e\right]^{*} \rightarrow \mathrm{GL}\left(n, p^{k}\right)$ be an isomorphism. Now He $4\left[\left(z_{p} G\right) e\right]^{*}$ and $p|| H e \mid$. But $p$ divides $\left|\operatorname{SL}\left(n, p^{k}\right)\right|([1]$, Theorem 4.11) and therefore $\mathrm{SL}\left(n, p^{k}\right)$ is not contained in $(H e) \theta$. Nor $\operatorname{can}(H e) \theta$ be contained in 
the centre of $\mathrm{GL}\left(n, p^{k}\right)$, for otherwise (He) $\theta$ would be in the centre of $M_{n}\left(\mathrm{GF}\left(p^{k}\right)\right)$, by [1], Theorem 4.8, and then $H e$ would be in the centre of $\left(z_{p} G\right) e$. It follows from Theorem 4.9 of [1] that $n=2, k=1$ and $p=2$ or 3 .

If $p=2$ then $(\mathrm{He}) \theta \subseteq \mathrm{GL}(2,2) \simeq S_{3}$. Since 2|| $\mathrm{Ge} \mid$, Ge must be abelian and $H e$ is in the centre of $G e$ and therefore of $\left(z_{p} G\right) e$, which is a contradiction.

Thus $p=3$. But the only normal subgroup of $\operatorname{GL}(2,3)$ which has order not divisible by 3 and which is not contained in the centre of $\mathrm{GL}(2,3)$ is isomorphic to the quaternion group of order 8 . But $H \triangleleft\left(z_{p} H\right) *$ and $p \backslash|H|$ so that $H$ (and therefore $H e$ ) must be abelian ([4], Theorem $]$ ), and we again have a contradiction.

\section{3. $p$ divides $|G|$ and $p \geq 3$}

Here $H$ is abelian ([4], Theorem 1 ).

LEMMA 3. If $p$ divides $|G|$ and $p \geq 3$ then $H$ is contained in the centre of $G$.

Proof. Firstly if $k \in G$ has order a power of $p$ we show that $k$ is in the centralizer of $H$. For let $h \in H$. Since $H$ is abelian we may assume that $k \notin H$. Because $(1+k)^{p^{n}}=1+k^{p^{n}}$ for all $n, 1+k$ is nilpotent and $l+(1+k)$ is a unit. Thus there exists $h^{\prime} \in H$ with $(2+k) h=h^{\prime}(2+k)$. We can equate the terms in $H$ to get $h^{\prime}=h$ and then $k h=h k$ as required.

Now suppose that $p|| H \mid$. If $g \in G$ we can write $g=g^{s} g^{t}$ where $g_{1}=g^{s}$ has order a power of $p$ and $g_{2}=g^{t}$ has order relatively prime to $p$. Then $L=H\left\langle g_{2}\right\rangle$ is a subgroup of $G$ and $p$ does not divide its order. Since $H \triangleleft\left(z_{p} L\right) *$ it follows from Lema 2 that $g_{2}$ centralizes $H$. Thus $g$ centralizes $H$.

Accordingly we may assume that $p$ divides $|H|$. In this case there 
exists $x \in H$ of order $p$. If $g \in G, h \in H$ and $n \geq 1$, then

$$
[(1-x) g]^{n}=(1-x)\left(1-g x g^{-1}\right) \ldots\left(1-g^{n-1} x g^{1-n}\right) g^{n} \text {. }
$$

Since $H \triangleleft G$ and $H$ is abelian it follows that $(1-x) g$ is nilpotent. Thus there exists $h^{\prime} \in H$ such that $[1+(1-x) g] h=h^{\prime}[1+(1-x) g]$. Again we can assume that $g \notin H$. A comparison of the terms in $H$ gives $h^{\prime}=h$ and then, since $p \geq 3$, the other terms yield $g h=h g$.

\section{4. $p=2$ and $|G|$ even}

Here either $H$ is abelian or $H \simeq S_{3}$ ([4], Theorem 1).

LEMMA 4. Suppose $p=2$ and $|G|$ is even. If $x \in G$ has order $2^{s}$ with $s \geq 2$ then $x$ is in the centralizer of $H$.

Proof. If $x \in H$ then $H$ must be abelian (as $S_{3}$ has no element of order 4 ) and so $x$ centralizes $H$. Thus we can assume that $x \notin H$. Since $\left(x+x^{-1}\right)^{2^{s}}=0,1+x+x^{-1}$ is a unit. Then if $h \in H$ there exists $h^{\prime} \in H$ with $\left(1+x+x^{-1}\right) h=h^{\prime}\left(1+x+x^{-1}\right)$. If we compare the terms in $H$ we have $h^{\prime}=h$ and the other terms then give $h^{-1} x h=x^{ \pm 1}$. But then $h$ is in the normalizer of $\langle x\rangle$ and so $(1+x) h$ is nilpotent which means that there exists $h^{\prime \prime} \in H$ with $[1+(1+x) h] h=h^{\prime \prime}[1+(1+x) h]$. If we compare the terms outside of $H$ we get $h^{\prime \prime}=x h x^{-1}$ and so $h+h^{2}=x h x^{-1}+x h x^{-1} h$. Now either $h=h^{2}$ or $h=x h x^{-1}$ or $h=x h x^{-1} h$, and we get $x h=h x$ in each case.

LEMMA 5. If $p=2,|G|$ is even and $H \simeq S_{3}$, then $G=H$.

Proof. We first show that if $z$ centralizes $H$ then $z=1$. For then $H(z)=H \times\langle z\rangle$ so that $z_{2}(H(z))$ is isomorphic to the group ring of $H$ over the ring $z_{2}\langle z\rangle$. Then, by Lemma $1, z=1$.

Now let $g \in G$. We can write $g=g_{1} g_{2}$ where $g_{1}$ has odd order and $g_{2}$ has order $2^{s} \quad(s \geq 0)$. We let

$H=\left\{a^{i} b^{j} \mid 0 \leq i \leq 1,0 \leq j \leq 2\right\}$ with $a^{2}=1=b^{3}$ and $b a=a b^{2}$. It 
is easy to see that $\langle b\rangle \triangleleft\left(z_{2} G\right)^{*}$ and so $(b) \triangleleft\left[z_{2}\left(\langle b\rangle\left\langle g_{1}\right\rangle\right)\right]^{*}$ and it follows from Lemma 2 that $g_{1} b=b g_{1}$. Also $g_{1} a g_{1}^{-1} \in H$ and has order 2 so that $g_{1} a g_{1}^{-1}=a b^{j}$ for some $j$ and then $\left(b^{-j} g_{1}\right) a=a\left(b^{-j} g_{1}\right)$. This means that $b^{-j} g_{1}$ centralizes $H$ and hence $g_{1} \in H$. If $s \geq 2, g_{2}$ centralizes $H$ (Lemma 4 ) which is a contradiction. Accordingly we can assume that $g_{2}$ has order 2 . If $x=g_{2} a g_{2}=g_{2} a g_{2}^{-1}$ then $x$ has order 2 and $g_{2} x g_{2}=a$. If $x=a, g_{2}$ centralizes $a$. If $x \neq a$ and if $w$ is the element of order 2 in $H$ distinct from $x$ and $a$ then $g_{2} w g_{2}^{-1}$ must equal $w$ and $g_{2}$ centralizes $w$. Since $g_{2}$ does not centralize $H$ we must have $g_{2} b \neq b g_{2}$ and so $g_{2} b=b^{2} g_{2}$. Then if $c$ is an element of order 2 in $H$ which $g_{2}$ centralizes, $\left(1+c+g_{2}\right)^{2}=1$ yet

$$
\left(1+c+g_{2}\right) b\left(1+c+g_{2}\right)=b+c b+c b^{2}+g_{2} b+g_{2} b^{2}
$$

which is not in $H$ unless $g_{2} \in H$. Thus $g \in H$ as required.

LEMMA 6. If $p=2,|G|$ is even and $H$ is abelian, then either $H$ is contained in the centre of $G$ or $H \simeq A_{3}$ and $G \simeq S_{3}$.

Proof. Suppose $g \in G \backslash H$ and $h \in H$. We can write $g=g_{1} g_{2}$ and $h=h_{1} h_{2}$ where $g_{1}$ and $h_{1}$ have odd order and $g_{2}$ and $h_{2}$ have order a power of 2 . Because $H$ is abelian, $\left(1+h_{2}\right) g$ is nilpotent and so there exists $h^{\prime} \in H$ with

$$
\left[1+\left(1+h_{2}\right) g\right] h_{2}=h^{\prime}\left[1+\left(1+h_{2}\right) g\right] \text {. }
$$

A comparison of the terms in $H^{\prime}$ yields $h^{\prime}=h_{2}$ and gives $g h_{2}+h_{2} g h_{2}=h_{2} g+h_{2}^{2} g$, from which we get $h_{2} g=g h_{2}$. If $K$ is the product of all the Sylow subgroups of $H$ of odd order then $K$ is a characteristic subgroup of $H$ and so $K \diamond\left(z_{2} G\right) *$. Since $L=K\left\langle g_{1}\right\rangle$ has 
odd order and $K \triangleleft\left(z_{2} L\right) *$ it follows from Lemma 2 that $g_{1}$ and $h_{1}$ commute. Thus it follows from Lemma 4 that $g$ and $h$ commite unless $g_{2}$ has order 2 and $g_{2} h_{1} \neq h_{1} g_{2}$.

Accordingly we examine what happens if there exist $x \in H$ of odd order and $z \in G \backslash H$. of order 2 with $x z \neq z x$. If $\gamma=(1+z) x(1+z)$ then $\gamma^{2}=0$ so that $\delta=1+\gamma$ is a unit of order 2 , which means that $\delta x \delta$ is in $H$ again. Because $H$ is abelian and normal in $G$ we have $z x^{i} z \cdot x^{j}=x^{j} \cdot z x^{i} z$ for all $i$ and $j$. A calculation then yields $\delta x \delta=x+x^{3}+z x z x^{2}+z x^{3} z+x z x^{2} z+x z x^{2}+z x^{2}+z x^{3}+x^{2} z x+x^{2} z+x^{3} z$. The terms not in $H$ must cancel out and this gives $x^{3}=1$ and $x z=z x^{2}$. (Note that $x^{2} z=z x^{2}$ is impossible as $x$ has odd order.)

We now assume that the centralizer of $H$ is not the whole of $G$. It follows from the above that there exist $b \in H$ of order 3 and $a \in G \backslash H$ of order 2 with $b a=a b^{2}$.

We first show that $H=\langle b\rangle$. For suppose that $b_{1} \in H \backslash(b)$. If $a b_{1} \neq b_{1} a$ then $a b_{1}=b_{1}^{2} a$ and $b_{1}^{3}=1$. But then if $\theta=(1+a) b(1+a)$, $\theta^{2}=0$ and $(1+\theta) b_{1}(1+\theta)$ is again in $H$, which leads to a contradiction. Hence $a b_{1}=b_{1} a$. Now if $\psi=(1+a)\left(b+b^{2}\right), \psi^{2}=0$ and so $\left(1+b_{1} \psi\right)^{2}=1$. Thus there exists $h^{\prime} \in H$ with $\left(1+b_{1} \psi\right) b=h^{\prime}\left(1+b_{1} \psi\right)$. The terms not in $H$ give $h^{\prime}=b^{-1}$ and then the terms in $(b)$ give a contradiction.

Suppose now that $g \in G$ is not in the centralizer of $H$. As before we can write $g=g_{1} g_{2}$ where $g_{1}$ has odd order and $g_{2}$ has order 2 , and we have shown that $b_{2} g=b^{2} g_{2}$. Suppose if possible that $a g_{2} k H$. If $\lambda=(1+a) b g_{2}(1+a)$ then $\lambda^{2}=0$, and so there exists $c \in H$ with $(1+\lambda) b=c(1+\lambda)$. If we compare the terms in $H$ we get $c=b$ and then 
the other terms show that $g_{2} a=b a g_{2}$. Since $g_{2} a=\left(a g_{2}\right)^{-1}$ is also not in $H$ we can interchange $g_{2}$ and $a$ and get $a g_{2}=b g_{2} a$, which yields $b^{2}=1$ and is a contradiction. Thus $a g_{2} \in H$ and $g_{2} \in a H$. Now $g_{1}$ commutes with $g_{2}$ (each is a power of $g$ ), $g_{1}$ commutes with $b$ and $a \in g_{2}(b)$ so that $g_{1}$ commutes with $a$. Also $g_{1} a=g\left(g_{2}^{-1} a\right) \in g^{H} \neq H$. Suppose if possible that $g_{1} k H$. If $n=(1+a)\left(b+b^{2}\right)$ then $n g_{1}=g_{1} n$ and $\left(g_{1} n\right)^{2}=0$, so there exists $a \in H$ with $\left(1+g_{1} n\right) b=d\left(1+g_{1} n\right)$. A comparison of the terms in $H$ gives $d=b$ and then the other terms yield $b=1$ which is a contradiction. Hence $g_{1} \in H$ and $g \in g_{2} H=a H$. If $H_{1}$ denotes the centralizer of $H$ we see that $a H_{1} \subseteq$ aH. Thus $H_{1}=H$ and $G=H \cup a H$ as required.

\section{References}

[1] E. Artin, Geometric algebra (Interscience, New York, London, 1957).

[2] Klaus E. Eldridge, "On normal subgroups in modular group algebras", (unpublished).

[3] J. Lambek, Lectures on rings and modules (Blaisdell, Waltham, Mass achusetts, 1966).

[4] K.R. Pearson, "On the units of a modular group ring", Bulz. Austral. Math. Soc. 7 (1972), 169-182.

Department of Mathematics,

La Trobe University,

Bundoora,

victoria. 